\title{
Spinning rough disc moving in a rarefied medium
}

\author{
By Alexander Plakhov ${ }^{1,2, *}$, Tatiana Tchemisova ${ }^{2}$ and Paulo Gouveia ${ }^{3}$ \\ ${ }^{1}$ University of Aberystwyth, Aberystwyth SY23 3BZ, Ceredigion, UK \\ ${ }^{2}$ University of Aveiro, Aveiro 3810-193, Portugal \\ ${ }^{3}$ Polytechnic Institute of Bragança, Bragança 5301-854, Portugal
}

We study the Magnus effect: deflection of the trajectory of a spinning body moving in a gas. It is well known that in rarefied gases, the inverse Magnus effect takes place, which means that the transversal component of the force acting on the body has opposite signs in sparse and relatively dense gases. The existing works derive the inverse effect from nonelastic interaction of gas particles with the body. We propose another (complementary) mechanism of creating the transversal force owing to multiple collisions of particles in cavities of the body surface. We limit ourselves to the two-dimensional case of a rough disc moving through a zero-temperature medium on the plane, where reflections of the particles from the body are elastic and mutual interaction of the particles is neglected. We represent the force acting on the disc and the moment of this force as functionals depending on 'shape of the roughness', and determine the set of all admissible forces. The disc trajectory is determined for several simple cases. The study is made by means of billiard theory, Monge-Kantorovich optimal mass transport and by numerical methods.

Keywords: inverse Magnus effect; free molecular flow; rough surface; billiards; optimal mass transport

\section{Introduction}

We are concerned here with the Magnus effect: the phenomenon governing deflection of the trajectory of spinning bodies (e.g. golf ball or football). Surprisingly enough, in highly rarefied media (on Mars or in the thin atmosphere at a height corresponding to low earth orbits, $150 \mathrm{~km}$ or more), the inverse effect takes place; this means that the trajectory deflection has opposite signs in sparse and in dense media.

There is a vast literature devoted to the Magnus effect, motivated by sports and technology applications (e.g. Prandtl 1926; Rubinov \& Keller 1961; Mehta 1985). The inverse effect is also well known to the physicists; study of this phenomenon becomes increasingly important nowadays because of potential applications to aerodynamics of artificial satellites (Wang 1972; Ivanov \& Yanshin 1980; Borg et al. 2003; Weidman \& Herczynski 2004; Borg \& Söderholm 2008). Theoretical studies on the inverse Magnus effect are based on models of non-elastic reflections of medium particles from convex bodies. 
Actually, all these models ignore roughness, which is always present on the body's surface. The kind of the roughness (that is, the shape of microscopic dimples, hollows, gullies, etc.) depends on the body material; the surface may also be artificially roughened. Owing to the roughness, particles bounce off the body surface in directions other than that prescribed by the visible orientation of the surface, and may also have multiple reflections.

We believe that roughness of the body surface should be incorporated in the model and propose a new approach to studying the Magnus effect. This approach is based on examining the shape of the body's cavities and is applied to a very idealized case of a two-dimensional rough disc, where all reflections are supposed to be elastic.

This approach meets evident difficulties: there is a huge variety of shapes governing the roughness. The existing literature deals with many different kinds of roughness: Gaussian, non-Gaussian, fractal, etc. Each of them provides the special kind of reflection law, which may be very hard to determine. The difficulty of the task seems to be immense.

Fortunately, there is an easier way to get rid of these difficulties. Instead of calculating the scattering law for each given roughness, a sort of inverse problem can be considered: determine the set of scattering laws for all possible shapes of roughness. The main tool for this approach has been developed by Plakhov (2009a). Having solved this problem, we are in a position to determine the main characteristic of the effect: the range of forces acting on the body and of the moments of these forces. Of relatively less importance, but quite illustrative, is the calculation of forces and trajectories for several special kinds of roughness.

We warn the reader against seeing this paper as directly applicable to reallife cases. Rather, it provides an insight into studying the cases of complex surfaces. The next steps in this way would be application of this approach to three-dimensional bodies and allowing for non-elastic reflections.

Another novelty of our paper consists in applying optimal mass transport (OMT), a very vivid and rapidly growing field of calculus of variations (see Rachev \& Rüschendorf (1998) and Villani (2003) for a review of the progress in this theory), to the study of the inverse Magnus effect. A sort of vectorvalued OMT problem naturally appears and is examined here. To the best of our knowledge, this kind of generalization of OMT has never been considered before.

This paper is a further development of the ideas briefly reported by Plakhov \& Tchemisova (2009).

We now proceed to a detailed description of the problem. A spinning twodimensional body moves through a homogeneous medium on the plane. The medium is extremely rarefied, so that the free path length of particles is much larger than the body's size. In such a case, the interaction of the body with the medium can be described in terms of free molecular flow, where point particles fall on the body's surface and each particle interacts with the body, but not with the other particles. There is no gravitation force. The particles of the medium remain at rest; that is, the absolute temperature of the medium equals zero. In a frame of reference moving forward together with the body, we have a parallel flow of particles falling on the body at rest (figure 1).

Neglecting the angular momentum of particles, each particle is identified with a mass point that approaches the body, makes several (maybe none) collisions with its surface and goes away. All reflections are perfectly elastic. 


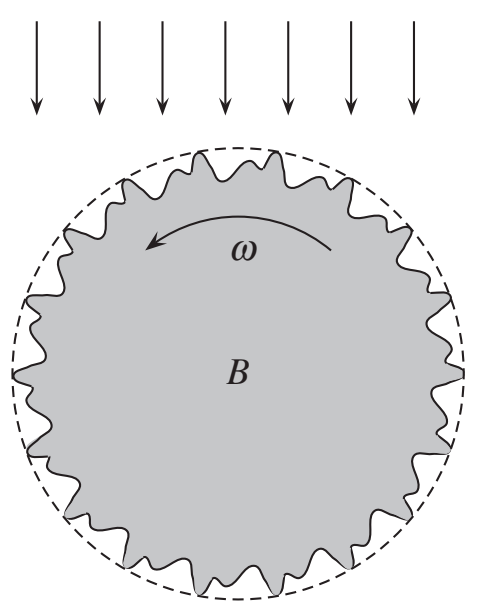

Figure 1. A rotating rough disc in a parallel flow of particles.

The body under consideration is a rough disc, that is, a set obtained from a circle by making infinitely small dimples on its boundary. More precisely, consider a sequence of sets $B_{m}, m=3,4,5, \ldots$, inscribed in the circle $B_{r}(O)$ of radius $r$ centred at a point $O$. Each set $B_{m}$ is invariant under the rotation by the angle $2 \pi / m$, and the intersection of $B_{m}$ with a certain $(2 \pi / m)$-sector $A_{m} O C_{m}$ formed by two radii $O A_{m}$ and $O C_{m}$ is a set bounded by these radii and by a piecewise smooth non-self-intersecting curve contained in the triangle $A_{m} O C_{m}$ and joining the points $A_{m}$ and $C_{m}$. These curves are similar for all $m .{ }^{1} \mathrm{~A}$ rough disc $B$ is associated with such a sequence of sets $B_{m}$. The curve is called the shape of roughness. All the values related to resistance or dynamics of the rough disc that are calculated below are understood as limits of the corresponding values for $B_{m}$ as $m \rightarrow \infty$.

Note in passing that the roughness introduced here is uniform: it is identical at each point of the circle boundary. In the case of non-uniform roughness, that is, if the shape of dimples varies along the boundary, periodical oscillations of the disc along the trajectory may happen, the period being equal to the period of one turn of the disc. The 'averaged' trajectory, however, coincides with the trajectory of the uniformly rough disc, where the roughness is obtained by 'averaging' the original one.

Denote by $\varphi(t)$ the rotation angle at the time $t$, by $\omega(t)$ the angular velocity of the disc, $\omega(t)=\mathrm{d} \varphi / \mathrm{d} t$, and let $\boldsymbol{v}(t)$ be the velocity of the disc centre of mass. Let us agree to measure the rotation angle and the angular velocity counterclockwise.

We consider the following problems: (A) determine the force of the medium resistance acting on the disc, find the moment of this force with respect to the disc centre of mass and investigate their dependence on the shape of roughness; (B) determine the set of admissible forces; and (C) analyse the motion of rough discs in the medium, that is, study the behaviour of the functions $\omega(t)$ and $\boldsymbol{v}(t)$. Problems (A) and (B) are primary with respect to $(\mathrm{C})$. In the paper, we will devote the main attention to problems (A) and (B), having just touched upon problem $(\mathrm{C})$, where we will restrict ourselves to deducing equations of motion and solving these equations for several simple cases.

\footnotetext{
${ }^{1}$ That is, one curve can be obtained from another one by a homothety and an isometry.
} 
(a)

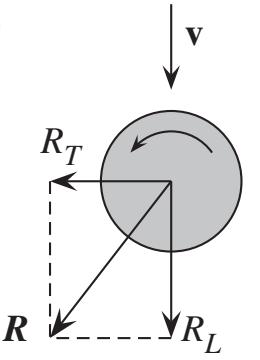

(b)

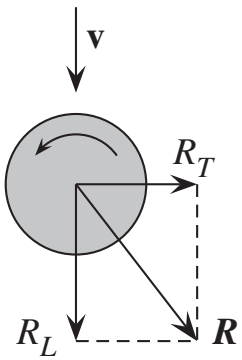

Figure 2. (a) The Magnus effect; (b) the inverse Magnus effect.

With each set $B_{m}$, we associate a distribution of mass inside $B_{m}$ such that the total mass $M$ is constant and the centre of the mass coincides with the centre $O$ of the set. We also assume that the moment of inertia $I_{m}$ of $B_{m}$ about $O$ converges to a positive value $I$ as $m \rightarrow \infty$. One always has $I \leq M r^{2}$. Denote $\beta=M r^{2} / I$; that is, $\beta$ is the inverse relative moment of inertia; we have $1 \leq \beta<+\infty$. In what follows, we will pay special attention to two particular cases: (i) $\beta=1$, the mass of the disc is concentrated near its boundary, and (ii) $\beta=2$, the mass is distributed uniformly in the disc.

The resistance force $\boldsymbol{R}_{m}\left(B_{m}, \varphi, \omega, \boldsymbol{v}\right)$ acting on the set $B_{m}$ and the moment of this force $R_{I, m}\left(B_{m}, \varphi, \omega, \boldsymbol{v}\right)$ depend on the shape of roughness, the rotation angle $\varphi$, the angular velocity $\omega$ and the velocity of translation $\boldsymbol{v}$. The equations of dynamics are

$$
M \frac{\mathrm{d} \boldsymbol{v}}{\mathrm{d} t}=\boldsymbol{R}_{m}\left(B_{m}, \varphi, \omega, \boldsymbol{v}\right), \quad I_{m} \frac{\mathrm{d} \omega}{\mathrm{d} t}=R_{I, m}\left(B_{m}, \omega, \boldsymbol{v}\right) \quad \text { and } \quad \frac{\mathrm{d} \varphi}{\mathrm{d} t}=\omega .
$$

Taking the limit $m \rightarrow \infty$, one gets the resistance force acting on the rough disc $\boldsymbol{R}(B, \omega, \boldsymbol{v})$ and the moment of this force $R_{I}(B, \omega, \boldsymbol{v})$, which do not depend on $\varphi$ anymore, and the equations for the disc dynamics take the form

$$
M \frac{\mathrm{d} \boldsymbol{v}}{\mathrm{d} t}=\boldsymbol{R}(B, \omega, \boldsymbol{v})
$$

and

$$
I \frac{\mathrm{d} \omega}{\mathrm{d} t}=R_{I}(B, \omega, v) .
$$

We shall see below that, generally speaking, $\boldsymbol{R}=\boldsymbol{R}(B, \omega, \boldsymbol{v})$ is not collinear to $v$.

If a transversal component of the resistance force appears, resulting in deflection of the body's trajectory, then we encounter the (proper or inverse) Magnus effect. If the direction of the transversal component coincides with the instantaneous velocity of the front point of the body, then a proper Magnus effect takes place. If these directions are opposite, then an inverse Magnus effect occurs (figure $2 a, b)$.

Note that the limiting case of slow rotation has been studied in detail by Plakhov $(2009 a, b)$. In that case, the mean resistance force is parallel to the direction of the body's motion, and therefore the Magnus effect does not appear. 


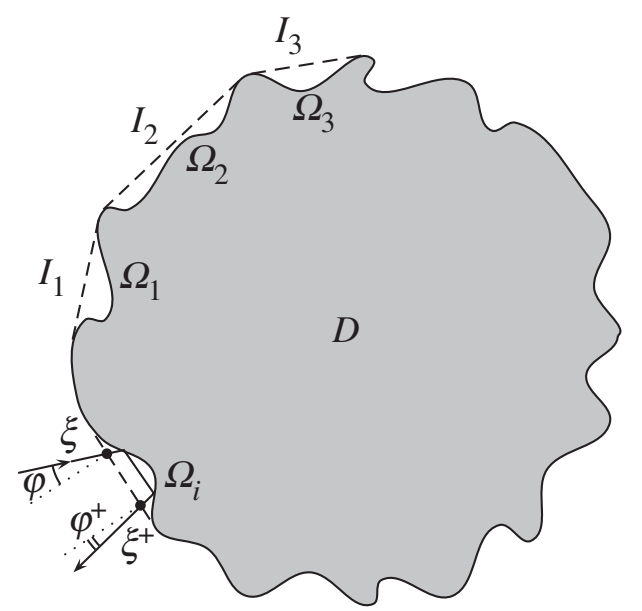

Figure 3. Cavities on the boundary of a non-convex set.

In the next section, to each rough disc $B$, we assign a measure $\nu_{B}$ characterizing the law of billiard scattering on $B$. The values $\boldsymbol{R}$ and $R_{I}$ are defined to be functions of the values $\nu_{B}, \omega$ and $\boldsymbol{v}$. In $\S 3$, we define the set of all possible values of $\boldsymbol{R}$, when $\omega$ and $\boldsymbol{v}$ are fixed and $\nu_{B}$ takes all admissible values. In other words, we answer the following question: what is the range of values of the force acting on a rough disc? While we look through all possible shapes of roughness, the vector $\boldsymbol{R}$ covers a fixed convex two-dimensional set. The problem of finding this set is formulated in terms of a special vector-valued Monge-Kantorovich problem and is solved numerically for several fixed values of the parameter $\lambda=\omega r / v$. Further, we calculate $\boldsymbol{R}$ and $R_{I}$ for some special values of $\nu_{B}$ (and thus for some special kinds of rough bodies). In $\S 4$, we deduce the equations of dynamics in a convenient form and solve them in several simple particular cases. Finally, in $\S 5$, a comparison of our results with those of the previous works on the inverse Magnus effect in rarefied media is given.

\section{Law of billiard scattering and resistance}

(a) Billiard scattering by a non-convex set

Let us first define the measure $\nu_{D}$ characterizing billiard scattering on a bounded simply connected set $D \subset \mathbb{R}^{2}$ with a piecewise smooth boundary. The set $\partial(\operatorname{conv} D) \backslash \partial D=\cup_{i \geq 1} I_{i}$ is the union of a finite or countable family of connected components $I_{i}, i=1,2, \ldots$ Each component $I_{i}$ is an open interval. (In figure 3, the dashed line denotes the intervals $I_{i}, i=1,2,3$.) Denote $I_{0}=\partial(\operatorname{conv} D) \cap \partial D$; in other words, $I_{0}$ is the 'convex part' of the boundary $\partial D$. Thus, $\partial D$ is the disjoint union $\partial D=\cup_{i \geq 0} I_{i}$.

Further, the set conv $D \backslash D$ is the union of a finite or countable collection of its connected components. For any $I_{i}$, there exists a set $\Omega_{i}$ from this collection such that $I_{i} \subset \partial \Omega_{i}$ (figure 3 ). The pair $\left(\Omega_{i}, I_{i}\right)$ will be called a cavity, and the interval $I_{i}$, the opening of the cavity.

Denote by $\boldsymbol{n}_{\xi}$ the outer unit normal to $\partial(\operatorname{conv} D)$ at the point $\xi \in \partial(\operatorname{conv} D)$. On the set $I_{i} \times[-\pi / 2, \pi / 2]$ with the coordinates $(\xi, \varphi)$, define the measure $\mu_{i}$ according to the formula $\mathrm{d} \mu_{i}=\cos \varphi \mathrm{d} \xi \mathrm{d} \varphi$, where $\mathrm{d} \xi$ and $\mathrm{d} \varphi$ stand for the 
one-dimensional Lebesgue measure. Consider the billiard in $\mathbb{R}^{2} \backslash D$. Fix $\xi \in I_{i}$ and $\varphi \in[-\pi / 2, \pi / 2]$, and take a billiard particle that starts moving at the point $\xi$ with the velocity forming the angle $\varphi$ with $-\boldsymbol{n}_{\xi}$. The particle makes one or several reflections at points of $\partial \Omega_{i} \backslash I_{i}$ and then intersects $I_{i}$ once again at the point $\xi^{+}=\xi_{i}^{+}(\xi, \varphi)$, the velocity at the moment of intersection forming the angle $\varphi^{+}=\varphi_{i}^{+}(\xi, \varphi)$ with the vector $\boldsymbol{n}_{\xi^{+}}, \varphi^{+} \in[-\pi / 2, \pi / 2]$. Notice that one always has $\boldsymbol{n}_{\xi}=\boldsymbol{n}_{\xi^{+}}$. In figure 3 , we have $\varphi<0$ and $\varphi^{+}=\varphi_{i}^{+}(\xi, \varphi)>0$.

Thus, for each $i$, we have defined the mapping $(\xi, \varphi) \mapsto\left(\xi_{i}^{+}, \varphi_{i}^{+}\right)$from a full measure subset of $I_{i} \times[-\pi / 2, \pi / 2]$ onto itself. This mapping is a bijection and involution, and preserves the measure $\mu_{i}$. In the particular case, where $i=0$, it holds $\xi_{0}^{+}=\xi$ and $\varphi_{0}^{+}=-\varphi$. Denote by $l_{i}=\left|I_{i}\right|$ the length of $I_{i}$, and by $l=\sum_{i>0} l_{i}=|\partial(\operatorname{conv} D)|$ the perimeter of conv $D$. Introduce the notation $\square:=[-\pi / 2, \pi / 2] \times[-\pi / 2, \pi / 2]$ and define the measures $\nu_{D}^{i}, i=0,1,2, \ldots$ on $\square$ as follows: $\nu_{D}^{i}(A):=\left(1 / l_{i}\right) \mu_{i}\left(\left\{(\xi, \varphi):\left(\varphi, \varphi_{i}^{+}(\xi, \varphi)\right) \in A\right\}\right)$ for any Borel set $A \subset \square$. In particular, the measure $\nu_{D}^{0}=: \nu_{0}$ is supported on the diagonal $\varphi^{+}=-\varphi$, and has the density $\mathrm{d} \nu_{0}\left(\varphi, \varphi^{+}\right)=\cos \varphi \cdot \delta\left(\varphi+\varphi^{+}\right)$. Finally, define $\nu_{D}:=1 / l \sum_{i \geq 0} l_{i} \nu_{D}^{i}$. The measure $\nu_{D}$ is called law of billiard scattering by $D$.

In a less formal way, the measure $\nu_{D}$ can be interpreted as follows. Place the body $D$ in a kind of 'ether' and keep it motionless. By ether, we mean a homogeneous isotropic medium composed of mutually non-interacting particles moving freely with unit velocity in all possible directions. When colliding with the body, the particles reflect elastically from its boundary. Thus, the particles of the ether behave like billiard particles in $\mathbb{R}^{2} \backslash D$.

For each particle that has reflected from $D$, fix the pair $\left(\varphi, \varphi^{+}\right)$of the angle of incidence $\varphi$ and the angle of reflection $\varphi^{+}$. The angle $\varphi$ is formed by the initial velocity of the particle and the vector $-\boldsymbol{n}_{\xi}$, whereas the angle $\varphi^{+}$is formed by its final velocity and the vector $\boldsymbol{n}_{\xi_{+}}$. Here, $\xi_{\text {and }} \xi^{+}$are the points of the first and second intersection of the particle's trajectory with $\partial(\operatorname{conv} D)$. The distribution of the set of pairs $\left(\varphi, \varphi^{+}\right)$for all particles that have collided with $D$ in a unit time period is described by the measure $\nu_{D}$.

Recall that given a Borel mapping $\pi: X \rightarrow Y$ of two sets $X \subset \mathbb{R}^{d_{1}}, Y \subset \mathbb{R}^{d_{2}}$, with the set $X$ being equipped with a Borel measure $\mu$, the so-called push-forward measure $\pi^{\#} \mu$ on $Y$ is defined by $\pi^{\#} \mu(A):=\mu\left(\pi^{-1}(A)\right)$ for any Borel set $A \subset Y$. Denote by $\pi_{\varphi}$ and $\pi_{\varphi^{+}}: \square \rightarrow[-\pi / 2, \pi / 2]$ the projections of the square $\square$ on its horizontal and vertical sides, respectively; $\pi_{\varphi}\left(\varphi, \varphi^{+}\right)=\varphi, \pi_{\varphi^{+}}\left(\varphi, \varphi^{+}\right)=\varphi^{+}$. The push-forward measures $\pi_{\varphi}^{\#} \nu$ and $\pi_{\varphi^{+}}^{\#} \nu$ are called marginal measures for $\nu$. They are defined on $[-\pi / 2, \pi / 2]$, and for any Borel set $A \subset[-\pi / 2, \pi / 2]$ we have $\pi_{\varphi}^{\#} \nu(A)=$ $\nu(A \times[-\pi / 2, \pi / 2]), \pi_{\varphi^{+}}^{\#} \nu(A)=\nu([-\pi / 2, \pi / 2] \times A)$.

Define the transformation of the square $\pi_{d}: \square \rightarrow \square$ exchanging the coordinates $\varphi$ and $\varphi^{+}$; that is, $\pi_{d}\left(\varphi, \varphi^{+}\right)=\left(\varphi^{+}, \varphi\right)$. The push-forward measure $\pi_{d}^{\#} \nu$ satisfies the condition $\pi_{d}^{\#} \nu(A)=\nu\left(\pi_{d}(A)\right)$ for any Borel set $A \subset \square$. In other words, the measures $\pi_{d}^{\#} \nu$ and $\nu$ are mutually symmetric with respect to the diagonal $\varphi=\varphi^{+}$.

Finally, define the measure $\gamma$ on $[-\pi / 2, \pi / 2]$ by $\mathrm{d} \gamma=\cos \varphi \mathrm{d} \varphi$, and denote by $\Pi^{\text {symm }}$ the set of measures $\nu$ on $\square$ satisfying the conditions

$$
\pi_{\varphi}^{\#} \nu=\gamma=\pi_{\varphi^{+}}^{\#} \nu \text { and } \pi_{d}^{\#} \nu=\nu .
$$




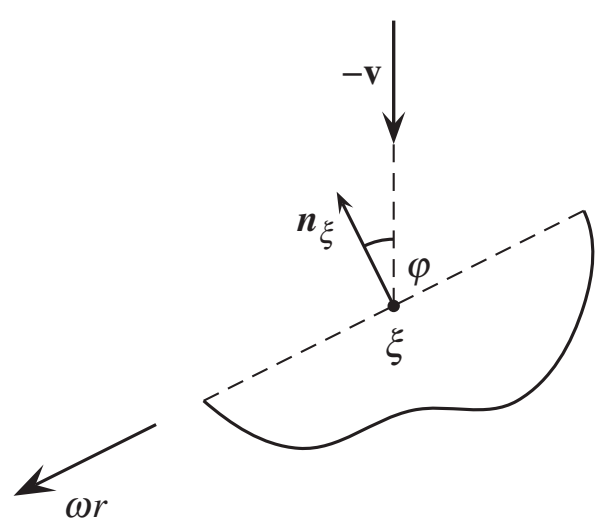

Figure 4. A particle falling on a cavity.

In other words, a generic measure from $\Pi^{\text {symm }}$ is symmetric with respect to the diagonal $\varphi=\varphi^{+}$, and both of its marginal measures coincide with $\gamma$. One has $\nu_{D}^{i} \in \Pi^{\mathrm{symm}}$; this can be easily deduced from the measure preserving and involutive properties of the mapping $(\xi, \varphi) \mapsto\left(\xi_{i}^{+}, \varphi_{i}^{+}\right)$; for details see Plakhov (2004). Hence, $\nu_{D} \in \Pi^{\text {symm }}$. Moreover, the following fundamental theorem characterizing the set of scattering laws holds true (Plakhov 2009a).

Theorem 2.1. Whatever the two sets $K_{1} \subset K_{2} \subset \mathbb{R}^{2}$ such that $\operatorname{dist}\left(\partial K_{1}, \partial K_{2}\right)>0$, the set of measures $\left\{\nu_{D}: K_{1} \subset D \subset K_{2}\right\}$ is everywhere dense in $\Pi^{\text {symm }}$ in the weak topology.

Now consider the rough disc $B$ generated by a sequence of sets $B_{m}$. All the cavities of all the sets $B_{m}$ are similar; therefore, $\nu_{B_{m}}$ does not depend on $m$ and we can set by definition $\nu_{B}:=\nu_{B_{m}}$. We will see later that the resistance of $B$ can be written down as a functional of $\nu_{B}$. The following theorem is obtained by a slight modification of the proof of theorem 2.1.

Theorem 2.2. Whatever $r>0$, the set $\left\{\nu_{B}: B\right.$ is a rough disc of radius $\left.r\right\}$ is everywhere dense in $\Pi^{\mathrm{symm}}$ in the weak topology.

\section{(b) Resistance of a rough disc}

Denote $v=|\boldsymbol{v}|$ and choose the (non-inertial) frame of reference $O x_{1} x_{2}$ such that the direction of the axis $O x_{2}$ coincides with the direction of the disc motion and the origin $O$ coincides with the disc centre. In this frame of reference, the disc stays at rest, and the flow of particles falls down on it at the velocity $-\boldsymbol{v}_{0}=(0 ;-\boldsymbol{v})^{\mathrm{T}}$. Here and in what follows, we represent vectors as columns; for instance, a vector $\boldsymbol{x}$ will be denoted by $\left[\begin{array}{l}x_{1} \\ x_{2}\end{array}\right]$ or $\left(x_{1} ; x_{2}\right)^{\mathrm{T}}$.

Let us calculate the force $\boldsymbol{R}$ of the medium resistance and the moment of this force $R_{I}$ with respect to $O$. To that end, first we consider the pre-limit body $B_{m}$. Parametrize the opening of each cavity by the variable $\xi$ varying from 0 to 1 (recall that all the cavities are identical). Denote by $\rho$ the flow density, by $\varphi$, the rotation angle of the cavity (that is, the external normal at the cavity opening equals $\left.\boldsymbol{n}_{\xi}=(-\sin \varphi ; \cos \varphi)^{\mathrm{T}}\right)$ and by $\boldsymbol{v}_{(m)}^{+}(\xi, \varphi)$, the final velocity of the particle entering the cavity at the point $\boldsymbol{\xi}$ with the velocity $-\boldsymbol{v}_{0}$ (figure 4 ). Note that 
$\Delta t=2 \pi /(\omega m)$ is the minimal time period between two identical positions of the rotating set $B_{m}$. Then the momentum imparted to $B_{m}$ by the particles of the flow during the time interval $\Delta t$ equals

$$
2 r \rho v \Delta t \int_{0}^{1} \int_{-\pi / 2}^{\pi / 2}\left(-\boldsymbol{v}_{0}-\boldsymbol{v}_{(m)}^{+}(\xi, \varphi)\right) \frac{1}{2} \cos \varphi \mathrm{d} \varphi \mathrm{d} \xi
$$

Consider the frame of reference $\tilde{O} \tilde{x_{1}} \tilde{x_{2}}$ having the centre at the midpoint of the cavity opening $I$; the axis $\tilde{O} \tilde{x_{1}}$ being parallel to $I$; and $\tilde{O} \tilde{x_{2}}$, codirectional with $\boldsymbol{n}_{\xi}$. That is, the frame of reference rotates jointly with the segment $I$. The change of variables from $\boldsymbol{x}=\left(x_{1} ; x_{2}\right)^{\mathrm{T}}$ to $\tilde{\boldsymbol{x}}=\left(\tilde{x_{1}} ; \tilde{x}_{2}\right)^{\mathrm{T}}$ and the inverse one are given by $\tilde{\boldsymbol{x}}=A_{-\omega t} \boldsymbol{x}-r \cos (\boldsymbol{\pi} / m) e_{\pi / 2}$ and $\boldsymbol{x}=A_{\omega t} \tilde{\boldsymbol{x}}+r \cos (\pi / m) e_{\pi / 2+\omega t}$, where $A_{\phi}=\left(\begin{array}{cc}\cos \phi & -\sin \phi \\ \sin \phi & \cos \phi\end{array}\right)$ and $\boldsymbol{e}_{\phi}=\left[\begin{array}{c}\cos \phi \\ \sin \phi\end{array}\right]$.

Suppose now that $\boldsymbol{x}(t)$ and $\tilde{\boldsymbol{x}}(t)$ are the coordinates of a moving point in the initial and rotating frames of reference, respectively, and let $\boldsymbol{v}=\left(v_{1} ; v_{2}\right)^{\mathrm{T}}=\mathrm{d} \boldsymbol{x} / \mathrm{d} t$ and $\tilde{\boldsymbol{v}}=\left(\tilde{v}_{1} ; \tilde{v}_{2}\right)^{\mathrm{T}}=\mathrm{d} \tilde{\boldsymbol{x}} / \mathrm{d} t$. Then,

$$
\tilde{\boldsymbol{v}}=A_{-\omega t} \boldsymbol{v}-\omega A_{\pi / 2-\omega t} \boldsymbol{x} \quad \text { and } \quad \boldsymbol{v}=A_{\omega t} \tilde{\boldsymbol{v}}+\omega A_{\pi / 2+\omega t} \tilde{\boldsymbol{x}}-\omega r \cos \left(\frac{\pi}{m}\right) \boldsymbol{e}_{\omega t} .
$$

We apply formulae (2.3) to the velocity of the particle at the two moments of its intersection with $I$. At the first moment, it holds $\omega t=\varphi$ and $\boldsymbol{x}=r e_{\pi / 2+\varphi}+o(1)$ as $m \rightarrow \infty$. (Here and in what follows, the estimates $o(1)$ are not necessarily uniform with respect to $\xi$ and $\varphi$.) Then the incidence velocity $-\boldsymbol{v}_{0}$ takes the form

$$
-\tilde{\boldsymbol{v}}_{0}=v(\lambda-\sin \varphi ;-\cos \varphi)^{\mathrm{T}}+o(1)=-v \varrho(-\sin x ; \cos x)^{\mathrm{T}}+o(1),
$$

where $\lambda=\omega r / v$ and

$$
\varrho=\varrho(\varphi, \lambda)=\sqrt{\lambda^{2}-2 \lambda \sin \varphi+1} \quad \text { and } \quad x=x(\varphi, \lambda)=\arcsin \frac{\lambda-\sin \varphi}{\varrho(\varphi, \lambda)} .
$$

As $m \rightarrow \infty$, the time spent by the particle in the cavity tends to zero; therefore, the rotating frame of reference can be considered 'approximately inertial' during that time, and the velocity at the second point of intersection is given by $\tilde{\boldsymbol{v}}^{+}=v \varrho(-\sin y ; \cos y)^{\mathrm{T}}+o(1)$, where $y=y(\xi, \varphi, \lambda)=\varphi^{+}(\xi, x(\varphi, \lambda))$. (Here $\xi^{+}(\xi, \varphi), \varphi^{+}(\xi, \varphi)$ denote the mapping generated by the cavity; $\S 2 a$ ) Applying the second formula in equation (2.3) and taking into account that $\tilde{\boldsymbol{x}}=o(1)$ and $\omega t=\varphi+o(1)$, we find the velocity in the initial frame of reference, $\boldsymbol{v}^{+}=\boldsymbol{v}_{(m)}^{+}(\xi, \varphi, \lambda)=v \varrho A_{\varphi}(-\sin y ; \cos y)^{\mathrm{T}}-v \lambda e_{\varphi}+o(1)=v^{+}(\xi, \varphi, \lambda)+o(1)$, where

$$
v^{+}(\xi, \varphi, \lambda)=v\left[\begin{array}{c}
-\varrho \sin (\varphi+y)-\lambda \cos \varphi \\
\varrho \cos (\varphi+y)-\lambda \sin \varphi
\end{array}\right] .
$$


Letting $m \rightarrow \infty$ in formulae (2.2) for the imparted momentum and dividing it by $\Delta t$, we get the following formula for the force of resistance acting on the disc:

$$
\boldsymbol{R}=\left[\begin{array}{l}
R_{T} \\
R_{L}
\end{array}\right]=r \rho v \int_{0}^{1} \int_{-\pi / 2}^{\pi / 2}\left(-\boldsymbol{v}_{0}-\boldsymbol{v}^{+}(\xi, \varphi, \lambda)\right) \cos \varphi \mathrm{d} \boldsymbol{g} \mathrm{d} \varphi .
$$

The angular momentum transmitted to $B_{m}$ by an individual particle equals $r v \varrho(\sin x+\sin y)+o(1)$ times the mass of the particle. Summing the angular momenta up over all incident particles and passing to the limit $m \rightarrow \infty$, one finds the moment of the resistance force acting on the disc,

$$
R_{I}=r^{2} \rho v \int_{0}^{1} \int_{-\pi / 2}^{\pi / 2} v \varrho(\varphi, \lambda)(\sin x(\varphi, \lambda)+\sin y(\xi, \varphi, \lambda)) \cos \varphi \mathrm{d} \xi \mathrm{d} \varphi .
$$

Theorem 2.3. The resistance and the moment of resistance of a rough disc of radius $r$ moving through a rarefied medium are equal to

$$
\boldsymbol{R}=\frac{8}{3} r \rho v^{2} \cdot \boldsymbol{R}\left[\nu_{B}, \lambda\right]
$$

and

$$
R_{I}=\frac{8}{3} r^{2} \rho v^{2} \cdot R_{I}\left[\nu_{B}, \lambda\right] .
$$

Here $\rho$ is the medium density, $v$ is the velocity of translation, $\omega$ is the angular velocity, $\lambda=\omega r / v$ and the dimensionless values $\boldsymbol{R}[\nu, \lambda]$ and $R_{I}[\nu, \lambda]$ are given by the integral formulas

$$
\boldsymbol{R}[\nu, \lambda]=\left[\begin{array}{l}
R_{T}[\nu, \lambda] \\
R_{L}[\nu, \lambda]
\end{array}\right]=\iint_{\square} \boldsymbol{c}(x, y, \lambda) \mathrm{d} \nu(x, y),
$$

and

$$
R_{I}[\nu, \lambda]=\iint_{\square} c_{I}(x, y, \lambda) \mathrm{d} \nu(x, y),
$$

with the functions $c$ and $c_{I}$ given by the relations (2.13)-(2.20). Recall that $\square=[-\pi / 2, \pi / 2] \times[-\pi / 2, \pi / 2]$. We also use the notation $\zeta=\zeta(x, \lambda)=$ $\arcsin \sqrt{1-\lambda^{2} \cos ^{2} x}$ and $x_{0}=x_{0}(\lambda)=\arccos 1 / \lambda ; \chi$ stands for the characteristic function.

(a) If $0<\lambda \leq 1$, then

$$
c(x, y, \lambda)=\frac{3}{4} \frac{(\lambda \sin x+\sin \zeta)^{3}}{\sin \zeta} \cos \frac{x-y}{2}\left[\begin{array}{c}
\cos \left(\zeta+\frac{x-y}{2}\right) \\
-\sin \left(\zeta+\frac{x-y}{2}\right)
\end{array}\right]
$$

and

$$
c_{I}(x, y, \lambda)=-\frac{3}{8} \frac{(\lambda \sin x+\sin \zeta)^{3}}{\sin \zeta}(\sin x+\sin y)
$$


and in particular,

$$
\boldsymbol{c}(x, y, 1)=3 \sin ^{2} x\left[\begin{array}{c}
\cos (2 x-y)+\cos x \\
-\sin (2 x-y)-\sin x
\end{array}\right] \chi_{x \geq 0}(x, y)
$$

and

$$
c_{I}(x, y, 1)=-3 \sin ^{2} x(\sin x+\sin y) \chi_{x \geq 0}(x, y) .
$$

In the limiting case $\lambda \rightarrow 0^{+}$, one has

$$
c(x, y, \lambda)=-\frac{3}{8}\left[\begin{array}{c}
\sin (x-y) \\
1+\cos (x-y)
\end{array}\right]+O(\lambda)
$$

and

$$
c_{I}(x, y)=\frac{9 \lambda}{8} \sin x(\sin x+\sin y)+O\left(\lambda^{2}\right)
$$

(b) If $\lambda>1$, then

$$
\begin{aligned}
c(x, y, \lambda)= & \frac{3}{2} \frac{\cos (x-y) / 2}{\sin \zeta}\left\{\left(\lambda^{3} \sin ^{3} x+3 \lambda \sin x \sin ^{2} \zeta\right) \cos \zeta\left[\begin{array}{c}
\cos \frac{x-y}{2} \\
-\sin \frac{x-y}{2}
\end{array}\right]\right. \\
& \left.-\left(3 \lambda^{2} \sin ^{2} x \sin \zeta+\sin ^{3} \zeta\right) \sin \zeta\left[\begin{array}{c}
\sin \frac{x-y}{2} \\
\cos \frac{x-y}{2}
\end{array}\right]\right\} \chi_{x \geq x_{0}}(x, y)
\end{aligned}
$$

and

$$
c_{I}(x, y, \lambda)=-\frac{3}{4} \frac{\lambda^{3} \sin ^{3} x+3 \lambda \sin x \sin ^{2} \zeta}{\sin \zeta}(\sin x+\sin y) \chi_{x \geq x_{0}}(x, y) .
$$

Proof. The theorem will be proved separately for cases $\lambda=1, \quad 0<\lambda<1$ and $\lambda>1$.

Case $\lambda=1$. We have $x=x(\varphi, 1)=\arcsin \sqrt{(1-\sin \varphi) / 2}=\pi / 4-\varphi / 2$, and so, the function $\varphi \mapsto x(\varphi, 1)$ is a bijection between the intervals $[-\pi / 2, \pi / 2]$ and $[0, \pi / 2]$. Further, one has $\varrho=\varrho(\varphi, 1)=\sqrt{2(1-\sin \varphi)}=2 \sin x, \cos \varphi=\sin 2 x$, and we get from equation (2.6) that $\boldsymbol{v}^{+}=v\left[\begin{array}{c}-2 \sin x \cos (2 x-y)-\sin 2 x \\ 2 \sin x \sin (2 x-y)-\cos 2 x\end{array}\right]$, wherefrom $-\boldsymbol{v}_{0}-\boldsymbol{v}^{+}=2 v \sin x\left[\begin{array}{c}\cos (2 x-y)+\cos x \\ -\sin (2 x-y)-\sin x\end{array}\right]$. Making the change of variables $\{\xi, \varphi\} \rightarrow\{\xi, x\}$ in the integral in equation (2.7) and using equation (2.9), one gets

$$
\boldsymbol{R}\left[\nu_{B}, 1\right]=3 \int_{0}^{1} \int_{0}^{\pi / 2} \sin ^{2} x\left[\begin{array}{c}
\cos (2 x-y)+\cos x \\
-\sin (2 x-y)-\sin x
\end{array}\right] \cos x \mathrm{~d} \xi \mathrm{d} x .
$$


In this integral, $y$ is the function of $\xi$ and $x, y=\varphi^{+}(\xi, x)$. Changing the variables once again, $\{\xi, x\} \rightarrow\{x, y\}$, and taking into account that $\cos x \mathrm{~d} \xi \mathrm{d} x=\mathrm{d} \nu_{B}(x, y)$, we obtain

$$
\boldsymbol{R}\left[\nu_{B}, 1\right]=3 \iint_{\square} \sin ^{2} x\left[\begin{array}{c}
\cos (2 x-y)+\cos x \\
-\sin (2 x-y)-\sin x
\end{array}\right] \mathrm{d} \nu_{B}(x, y) .
$$

Here the symbol $\square$ stands for the rectangle $x \in[0, \pi / 2], y \in[-\pi / 2, \pi / 2]$.

The moment of the resistance force is calculated analogously, resulting in

$$
\begin{aligned}
R_{I}\left[\nu_{B}, 1\right] & =-3 \int_{0}^{1} \int_{0}^{\pi / 2} \sin ^{2} x(\sin x+\sin y) \cos x \mathrm{~d} \xi \mathrm{d} x \\
& =-3 \iint_{\square} \sin ^{2} x(\sin x+\sin y) \mathrm{d} \nu_{B}(x, y) .
\end{aligned}
$$

Case $0<\lambda<1$. The second relation in equation (2.5) implies that for a fixed value of $\lambda, x=x(\varphi, \lambda)$ is a monotone decreasing function of $\varphi$ that varies from $\pi / 2$ to $-\pi / 2$ as $\varphi$ changes from $-\pi / 2$ to $\pi / 2$. From formulae (2.4) and the first relation in equation (2.5), we have $\sin \varphi=\lambda \cos ^{2} x-\sin x \sqrt{1-\lambda^{2} \cos ^{2} x}, \cos \varphi=$ $\cos x\left(\lambda \sin x+\sqrt{1-\lambda^{2} \cos ^{2} x}\right), \varrho=\lambda \sin x+\sqrt{1-\lambda^{2} \cos ^{2} x}$. Recall that

$$
\zeta=\zeta(x, \lambda)=\arcsin \sqrt{1-\lambda^{2} \cos ^{2} x}
$$

one has $\cos \zeta=\lambda \cos x, x+\zeta=\pi / 2-\varphi, \zeta \in[\arccos \lambda, \pi / 2]$, and taking into account equation (2.6), we get $-\boldsymbol{v}_{0}-\boldsymbol{v}^{+}=v(\lambda \sin x+\sin \zeta) \cdot 2 \cos (x-y) / 2$ $\left[\begin{array}{c}\cos (\zeta+(x-y) / 2) \\ -\sin (\zeta+(x-y) / 2)\end{array}\right], \cos \varphi / \cos x=\lambda \sin x+\sin \zeta=\varrho$ and $\mathrm{d} \varphi / \mathrm{d} x=-1-\mathrm{d} \zeta / \mathrm{d} x$ $=-(\lambda \sin x+\sin \zeta) / \sin \zeta$.

Using the obtained formula, making the change of variables $\{\xi, \varphi\} \rightarrow\{\xi, x\}$ in the integral (2.7), and taking into account equation (2.9), one gets

$$
\boldsymbol{R}\left[\nu_{B}, \lambda\right]=\frac{3}{4} \int_{0}^{1} \int_{-\pi / 2}^{\pi / 2} \frac{(\lambda \sin x+\sin \zeta)^{3}}{\sin \zeta} \cos \frac{x-y}{2}\left[\begin{array}{c}
\cos \left(\zeta+\frac{x-y}{2}\right) \\
-\sin \left(\zeta+\frac{x-y}{2}\right)
\end{array}\right] \cos x \mathrm{~d} \xi \mathrm{d} x .
$$

Finally, the change of variables $\{\xi, x\} \rightarrow\{x, y\}$ results in

$$
\boldsymbol{R}\left[\nu_{B}, \lambda\right]=\frac{3}{4} \iint_{\square} \frac{(\lambda \sin x+\sin \zeta)^{3}}{\sin \zeta} \cos \frac{x-y}{2}\left[\begin{array}{c}
\cos \left(\zeta+\frac{x-y}{2}\right) \\
-\sin \left(\zeta+\frac{x-y}{2}\right)
\end{array}\right] \mathrm{d} \nu_{B}(x, y) .
$$

Recall that the symbol $\square$ denotes the square $[-\pi / 2, \pi / 2] \times[-\pi / 2, \pi / 2]$ and $\zeta=\zeta(x, \lambda)$. 
In a similar way, from equation (2.8), one gets

$$
R_{I}=-\frac{3}{8} \int_{0}^{1} \int_{-\pi / 2}^{\pi / 2} \frac{(\lambda \sin x+\sin \zeta)^{3}}{\sin \zeta}(\sin x+\sin y) \cos x \mathrm{~d} \xi \mathrm{d} x
$$

wherefrom

$$
R_{I}\left[\nu_{B}, \lambda\right]=-\frac{3}{8} \iint_{\square} \frac{(\lambda \sin x+\sin \zeta)^{3}}{\sin \zeta}(\sin x+\sin y) \mathrm{d} \nu_{B}(x, y) .
$$

Formulae (2.21) and (2.22) are the particular cases of equations (2.24) and (2.25) for $\lambda=1$. This can be easily verified taking into account that $\zeta(x, 1)=|x|$.

Case $\lambda>1$. In this case, $x=x(\varphi, \lambda)$ equation (2.5) is not injection any more. When $\varphi$ varies from $-\pi / 2$ to $\varphi_{0}=\varphi_{0}(\lambda):=\arcsin 1 / \lambda$, the value of $x$ monotonically decreases from $\pi / 2$ to $x_{0}=x_{0}(\lambda)=\arccos 1 / \lambda$, and when $\varphi$ varies from $\varphi_{0}$ to $\pi / 2$, $x$ monotonically increases from $x_{0}$ to $\pi / 2$. Denote by $\varphi_{-}:=\varphi_{-}(x, \lambda)$ and $\varphi_{+}:=$ $\varphi_{+}(x, \lambda)$ the functions inverse to $x(\varphi, \lambda)$ on the intervals $\left[-\pi / 2, \varphi_{0}\right]$ and $\left[\varphi_{0}, \pi / 2\right]$, respectively. Then, one has $\sin \varphi_{ \pm}=\lambda \cos ^{2} x \pm \sin x \sqrt{1-\lambda^{2} \cos ^{2} x}$.

Here and in what follows, the signs ' + ' and ' - ' are related to the functions $\varphi_{+}$and $\varphi_{-}$respectively. The values $\varphi_{+}, \varphi_{-}$and $\zeta=\zeta(x, \lambda)$ equation (2.23) satisfy the relations $\pi / 2-\varphi_{+}=x-\zeta, \pi / 2-\varphi_{-}=x+\zeta$. The function $\zeta$ is defined for $x \in\left[x_{0}, \pi / 2\right]$ and monotonically increases from 0 to $\pi / 2$, when $x$ changes in the interval $\left[x_{0}, \pi / 2\right]$.

After some algebra, one gets $\cos \varphi_{ \pm} / \cos x=\sin (x \mp \zeta) / \cos x=\lambda \sin x \mp$ $\sin \zeta ; \quad \pm \mathrm{d} \varphi_{ \pm} / \mathrm{d} x=\mathrm{d} \zeta / \mathrm{d} x \mp 1=\lambda \sin x \mp \sin \zeta / \sin \zeta ; \quad \varrho_{ \pm}=\lambda \sin x \mp \sin \zeta ; \quad-\boldsymbol{v}_{0}-$ $\boldsymbol{v}_{ \pm}^{+}=v(\lambda \sin x \mp \sin \zeta) \cdot 2 \cos (x-y) / 2\left[\begin{array}{c}\cos ((x-y) / 2 \mp \zeta) \\ -\sin ((x-y) / 2 \mp \zeta)\end{array}\right]$. Here the shorthand notation $\varrho_{ \pm}=\varrho\left(\varphi_{ \pm}(x, \lambda), \lambda\right), \quad \boldsymbol{v}_{ \pm}^{+}=\boldsymbol{v}^{+}\left(\xi, \varphi_{ \pm}(x, \lambda), \lambda\right), \quad y=y\left(\xi, \varphi_{ \pm}(x, \lambda), \lambda\right)=$ $\varphi_{i}^{+}(\xi, x)$ is used.

The resistance force takes the form $\boldsymbol{R}\left[\nu_{B}, \lambda\right]=\boldsymbol{R}_{-}+\boldsymbol{R}_{+}$, where

$$
\begin{aligned}
\boldsymbol{R}_{ \pm} & =\frac{3}{8} \int_{0}^{1} \int_{x_{0}}^{\pi / 2}\left(-\boldsymbol{v}_{0}-\boldsymbol{v}_{ \pm}^{+}\right) \frac{\cos \varphi_{ \pm}}{\cos x}\left( \pm \frac{\mathrm{d} \varphi_{ \pm}}{\mathrm{d} x}\right) \cos x \mathrm{~d} \xi \mathrm{d} x \\
& =\frac{3}{4} \int_{0}^{1} \int_{x_{0}}^{\pi / 2} \frac{(\lambda \sin x \mp \sin \zeta)^{3}}{\sin \zeta} \cos \frac{x-y}{2}\left[\begin{array}{c}
\cos \left(\frac{x-y}{2} \mp \zeta\right) \\
-\sin \left(\frac{x-y}{2} \mp \zeta\right)
\end{array}\right] \cos x \mathrm{~d} \xi \mathrm{d} x .
\end{aligned}
$$

Summing the integrals above and making the change of variables, one obtains

$$
\begin{aligned}
\boldsymbol{R}\left[\nu_{B}, \lambda\right]= & \frac{3}{2} \iint_{\square} \frac{\cos (x-y) / 2}{\sin \zeta}\left\{\left(\lambda^{3} \sin ^{3} x+3 \lambda \sin x \sin ^{2} \zeta\right) \cos \zeta\left[\begin{array}{c}
\cos \frac{x-y}{2} \\
-\sin \frac{x^{2}-y}{2}
\end{array}\right]\right. \\
& \left.-\left(3 \lambda^{2} \sin ^{2} x \sin \zeta+\sin ^{3} \zeta\right) \sin \zeta\left[\begin{array}{l}
\sin \frac{x-y}{2} \\
\cos \frac{x-y}{2}
\end{array}\right]\right\} \mathrm{d} \nu_{B}(x, y) .
\end{aligned}
$$

Here the symbol $\square$ stands for the rectangle $\left[x_{0}, \pi / 2\right] \times[-\pi / 2, \pi / 2]$. 
The moment of the resistance force is calculated analogously. One has $R_{I}\left[\nu_{B}, \lambda\right]=R_{I-}+R_{I+}$, where

$$
\begin{aligned}
R_{I \pm} & =-\frac{3}{8} \int_{0}^{1} \int_{x_{0}}^{\pi / 2} \varrho_{ \pm} \frac{\cos \varphi_{ \pm}}{\cos x}\left( \pm \frac{\mathrm{d} \varphi_{ \pm}}{\mathrm{d} x}\right)(\sin x+\sin y) \cos x \mathrm{~d} \xi \mathrm{d} x \\
& =-\frac{3}{8} \int_{0}^{1} \int_{x_{0}}^{\pi / 2} \frac{(\lambda \sin x \mp \sin \zeta)^{3}}{\sin \zeta}(\sin x+\sin y) \cos x \mathrm{~d} \xi \mathrm{d} x .
\end{aligned}
$$

Therefore,

$$
R_{I}\left[\nu_{B}, \lambda\right]=-\frac{3}{4} \int_{0}^{1} \int_{x_{0}}^{\pi / 2} \frac{\lambda^{3} \sin ^{3} x+3 \lambda \sin x \sin ^{2} \zeta}{\sin \zeta}(\sin x+\sin y) \cos x \mathrm{~d} \xi \mathrm{d} x .
$$

Making the change of variables, we have

$$
R_{I}\left[\nu_{B}, \lambda\right]=-\frac{3}{4} \iint_{\square} \frac{\lambda^{3} \sin ^{3} x+3 \lambda \sin x \sin ^{2} \zeta}{\sin \zeta}(\sin x+\sin y) \mathrm{d} \nu_{B}(x, y) .
$$

The theorem is proved.

\section{Magnus effect}

We are primarily concerned here with determining the two-dimensional set of admissible normalized forces $\mathcal{R}_{\lambda}:=\left\{\boldsymbol{R}[\nu, \lambda]: \nu \in \Pi^{\mathrm{symm}}\right\}$.

Recall that according to the classification theorem 2.1, for each $\boldsymbol{R} \in \mathcal{R}_{\lambda}$ there exists a disc equipped with a suitable cavity that experiences a force arbitrarily close to $\boldsymbol{R}$ when moving at the relative angular velocity $\lambda$. However, this theorem gives us no idea how this cavity looks. It may well be too complicated to appear in nature or be fabricated. Therefore, it makes sense to describe subsets of $\mathcal{R}_{\lambda}$ generated by simple shapes. In this section, we present subsets generated by triangles and their combinations. Besides, we calculate analytically the resistance force and its moment for several simple shapes of cavity (rectangle, right isosceles triangle, etc.).

\section{(a) Vector-valued Monge-Kantorovich problem}

Here, we determine the set of all possible resistance forces that can act on a rough disc, with fixed angular velocity. The force is scaled so that the resistance of the 'ordinary circle' equals $(0 ;-1)^{\mathrm{T}}$. The problem is as follows: given $\lambda$, find the two-dimensional set

$$
\mathcal{R}_{\lambda}=\left\{\boldsymbol{R}[\nu, \lambda]: \nu \in \Pi^{\mathrm{symm}}\right\} .
$$

It can be viewed as a restriction of the following more general problem: find the three-dimensional set

$$
\left\{\left(\boldsymbol{R}[\nu, \lambda] ; R_{I}[\nu, \lambda]\right): \nu \in \Pi^{\mathrm{symm}}\right\} .
$$

The latter problem is more important, but also more time-consuming, and is mainly postponed to the future. The only exception is the case $\lambda=1$, where several 'level sets' $\mathcal{R}_{1, c}=\left\{\boldsymbol{R}[\nu, 1]: \nu \in \Pi^{\mathrm{symm}}, R_{I}[\nu, 1]=c\right\}$ are depicted in figure 5 , 

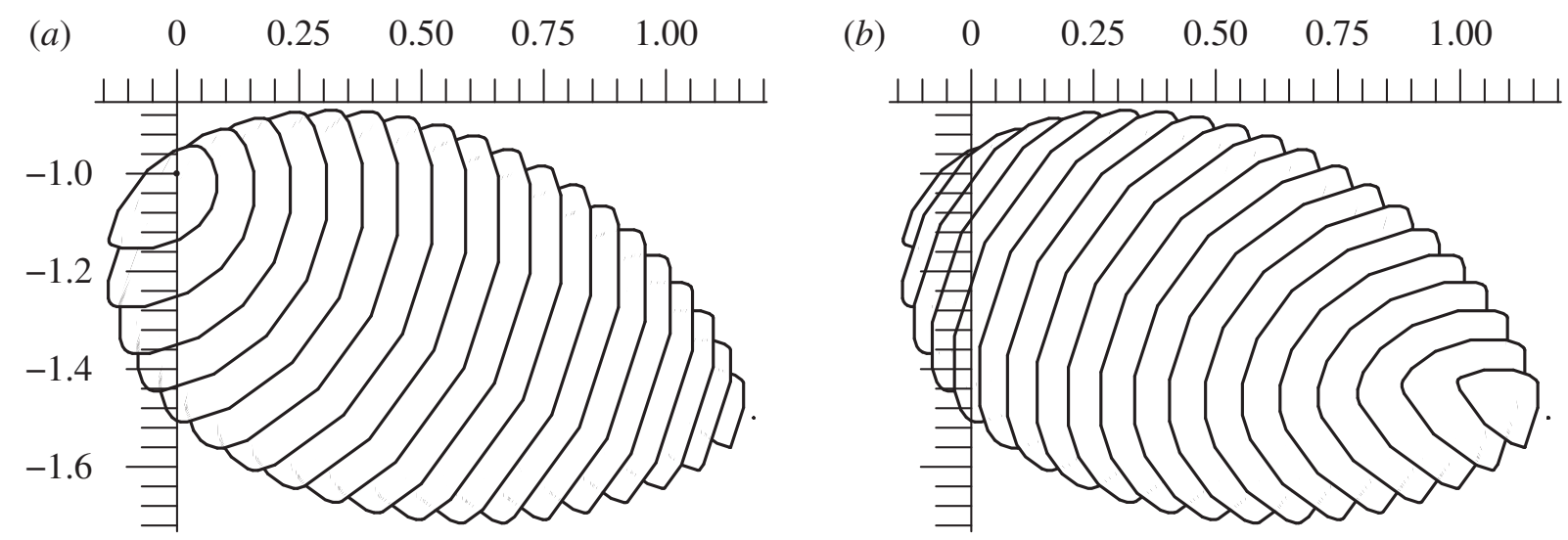

Figure 5. The 'level sets' $\mathcal{R}_{1, c}=$ all possible values of $\boldsymbol{R}[\nu, 1]$, with $\left.R_{I}[\nu, 1]=c\right\}$ are shown for 21 values of $c$, from left to right: $c=0,-0.075,-0.15,-0.225, \ldots,-1.425,-1.5$. (a) 'View from above' and $(b)$ 'view from below' on these sets.

suggesting what the corresponding three-dimensional set looks like. In this case, $R_{I}[\nu, 1]$ varies between -1.5 and 0 , and the level sets are found for 21 values $c=-1.5,-1.425,-1.35, \ldots,-0.15,-0.075,0$.

Note that the functional $\boldsymbol{R}$, defined on the set $\Pi^{\text {symm }}$ by formulae $(2.11)$, will not change if the integrand $c$ is replaced with the symmetrized function $\boldsymbol{c}^{\mathrm{symm}}(x, y, \lambda)=(1 / 2)(\boldsymbol{c}(x, y, \lambda)+\boldsymbol{c}(y, x, \lambda)): \quad \boldsymbol{R}[\nu, \lambda]=\iint_{\square} \boldsymbol{c}^{\mathrm{symm}}(x, y, \lambda) \mathrm{d} \nu(x, y)$. Denote by $\Pi$ the set of measures $\nu$ on the square $\square$ that satisfy the first condition in equation (2.1), that is, the set of measures with both marginals equal to $\gamma$. For any $\nu \in \Pi$, it holds $\iint_{\square} \boldsymbol{c}^{\text {symm }} \mathrm{d} \nu=\iint_{\square} \boldsymbol{c}^{\text {symm }} \mathrm{d} \nu^{\text {symm }}$, where $\nu^{\text {symm }}=$ $(1 / 2)\left(\nu+\pi_{d}^{\#} \nu\right) \in \Pi^{\text {symm }}$. It follows that

$$
\mathcal{R}_{\lambda}=\left\{\iint_{\square} c^{\mathrm{symm}}(x, y, \lambda) \mathrm{d} \nu(x, y): \nu \in \Pi\right\} .
$$

The problem of finding $\mathcal{R}_{\lambda}$ in equation (3.2) is a vector-valued analogue of the Monge-Kantorovich problem. The difference consists in the fact that the cost function, and therefore the functional, are vector-valued. The set $\mathcal{R}_{\lambda}$ is convex, since it is the image of the convex set $\Pi$ under a linear mapping.

Note that, owing to formulae $(2.17), \boldsymbol{c}^{\mathrm{symm}}\left(x, y, 0^{+}\right)=(3 / 8)(1+\cos (x-y))$ $(0 ; 1)^{\mathrm{T}}$; therefore, the problem of finding $\mathcal{R}_{0^{+}}$amounts to minimizing and maximizing the integral $3 / 8 \iint_{\square}(1+\cos (x-y)) \mathrm{d} \nu(x, y)$ over all $\nu \in \Pi$. This special Monge-Kantorovich problem was solved by Plakhov (2004); the minimal and maximal values of the integral were found to be $0.9878 \ldots$ and 1.5 .

In figures 6 and 7 , we present numerical solutions of this problem for the values $\lambda=0.1,0.3$ and 1 , as well as the analytical solution for $\lambda=0^{+}$. The case of larger $\lambda$ requires more involved calculation and therefore is postponed to the future. The method of solution is the following: for $n$ equidistant vectors $\boldsymbol{e}_{i}, i=1, \ldots, n$, on $S^{1}$, we find the solution of the Monge-Kantorovich problem $\inf \left\langle\boldsymbol{R}[\nu, \lambda], \boldsymbol{e}_{i}\right\rangle=: r_{i}$. Here $\langle\cdot, \cdot\rangle$ stands for the scalar product. This problem is reduced to the transport problem of linear programming and is solved numerically. ${ }^{2}$

${ }^{2}$ All the computational tests were performed on a PC Pentium IV, $2.0 \mathrm{GHz}$ and $512 \mathrm{Mb}$ RAM and using the optimization package XPRESS-IVE, v. 1.19.00 with the modeller MOSEL. 


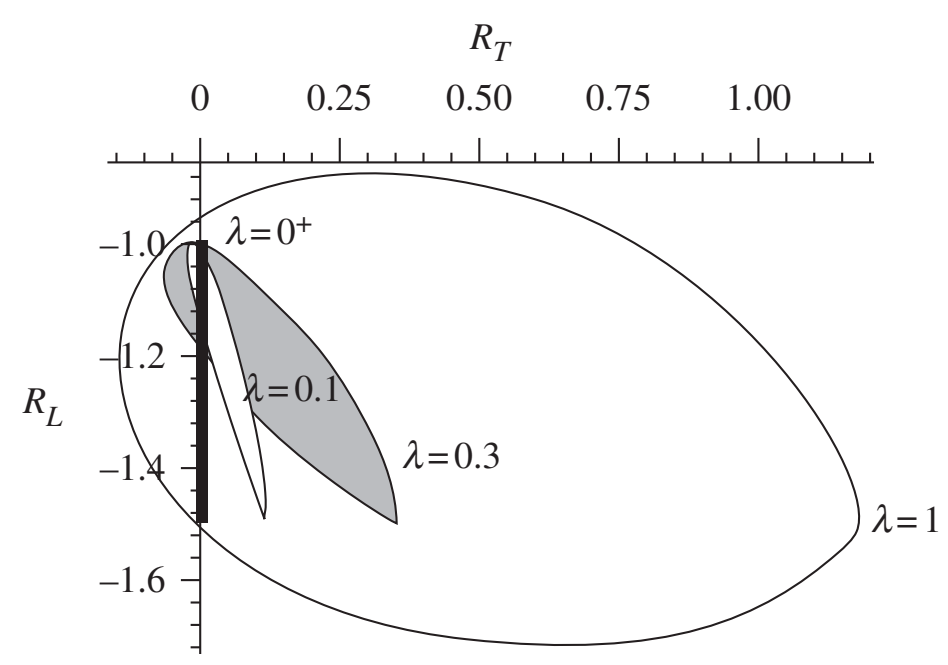

Figure 6 . The convex sets $\mathcal{R}_{\lambda}$ with $\lambda=0^{+}, 0.1,0.3$ and 1 are shown. The set $\mathcal{R}_{0^{+}}$is the vertical segment with the endpoints $(0,-0.9878 \ldots)$ and $(0,-1.5)$.

Next, the intersection of the half-planes $\left\langle\boldsymbol{r}, \boldsymbol{e}_{i}\right\rangle \geq r_{i}$ is built. It is a convex polygon approximating the required set $\mathcal{R}_{\lambda}$, and the approximation accuracy increases as $n$ increases. The value $n=100$ was used in our calculations.

In figure 6 , the sets $\mathcal{R}_{\lambda}$ are shown for $\lambda=0^{+}, 0.1,0.3$ and 1 . The set $\mathcal{R}_{0^{+}}$is the vertical segment $\{0\} \times[-1.5,-0.9878], \mathcal{R}_{0.1}$ is the thin set with white interior and $\mathcal{R}_{0.3}$ is the set with grey interior. The largest set is $\mathcal{R}_{1}$.

In figure 7 , the same sets are shown in more detail. In figure $7 b-d$, additionally, we present the regions corresponding to all possible kinds of roughness related to triangular cavities (and to cavities formed by combinations of different triangles), with the angles being multiples of $5^{\circ}$. These regions are coloured grey. For $\lambda=0^{+}$, the corresponding region is the vertical interval $\{0\} \times[-1.42,-1]$ marked by a (slightly shifted) dashed line in figure $7 a$.

The part of the set $\mathcal{R}_{\lambda}$ situated to the left of the vertical axis corresponds to resistance forces producing the proper Magnus effect. The part of $\mathcal{R}_{\lambda}$ to the right of this axis is related to forces that cause the inverse Magnus effect. We can see that the majority of the set (in the case $\lambda=1$, approx. $93.6 \%$ of the area) is situated to the right of the axis. This suggests that the inverse effect is a more common phenomenon than the proper one. Actually, although theorem 2.2 guarantees the existence of an everywhere dense subset of $\mathcal{R}_{\lambda}$ generated by shapes of roughness, we never encountered a shape producing the proper Magnus effect (and thus corresponding to a point on the left of the vertical axis).

\section{(b) Special cases of rough discs}

We present here only the final expressions for the forces and their moments calculated by formula (2.13)-(2.20) from theorem 2.3; the calculation details are omitted.

(1) Circle (no cavities). The measure $\nu_{0}$ corresponding to the circle is given by $\mathrm{d} \nu_{0}(x, y)=\cos x \cdot \delta(x+y)$. One has $R_{T}\left[\nu_{0}, \lambda\right]=R_{I}\left[\nu_{0}, \lambda\right]=0$ and $R_{L}\left[\nu_{0}, \lambda\right]=-1$. Thus, as one could expect, the resistance does not depend on the angular velocity and is collinear to the body's velocity. There is no Magnus effect in this case. 


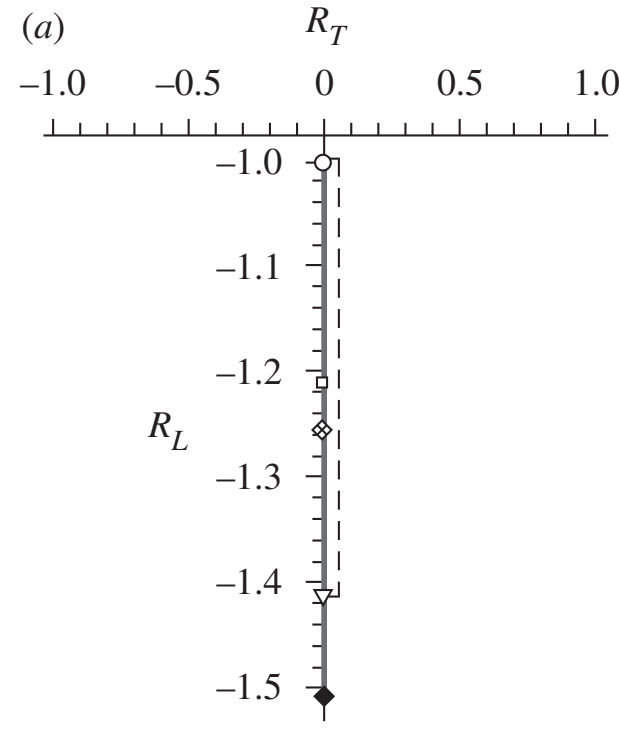

(c)

$$
R_{T}
$$
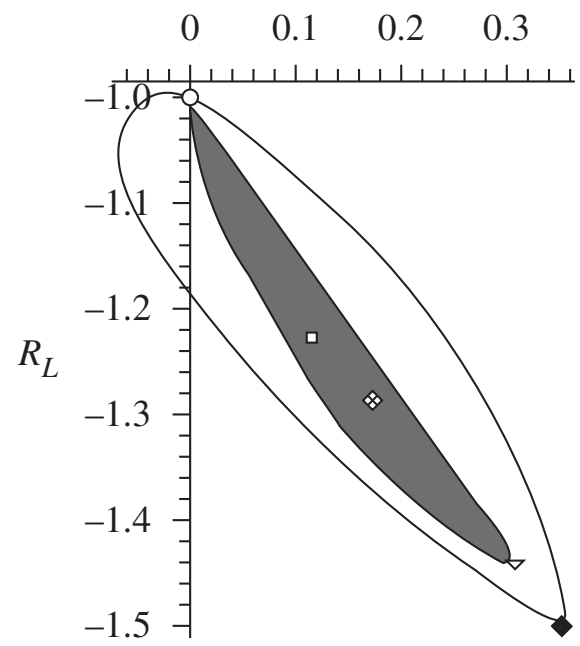

(b) $\quad R_{T}$

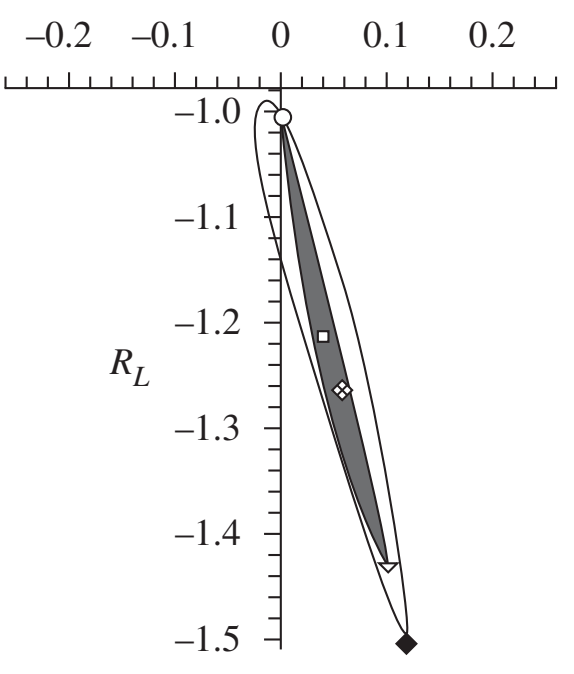

$(d)$

$$
R_{T}
$$

$$
\begin{array}{lllll}
0 & 0.25 & 0.50 & 0.75 & 1.00
\end{array}
$$

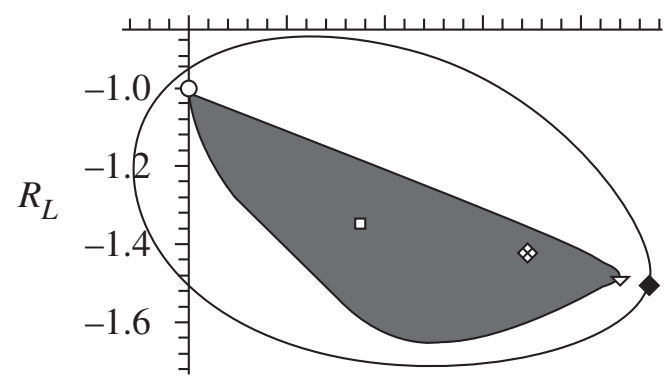

Figure 7 . The sets $\mathcal{R}_{\lambda}$ with $(a) \lambda=0^{+},(b) 0.1,(c) 0.3$ and $(d) 1$ are shown here separately. The values $R[\nu, \lambda]$, with $\nu=\nu_{0}, \nu_{\star}, \nu_{\text {rect }}, \nu_{\nabla}, \nu_{\otimes}$ are indicated by the symbols open circle, filled diamond, open square, inverse triangle and circumscribed cross, respectively. ( $a$ ) The region generated by triangular cavities is marked by a (slightly shifted) vertical dashed line. It is the interval with the endpoints $(0,-1)$ and $(0,-1.42) .(b-d)$ The regions generated by triangular cavities are painted over.

(2) Retroreflector. There exists a unique measure $\nu_{\star} \in \Pi^{\mathrm{symm}}$ supported on the diagonal $x=y$; its density equals $\mathrm{d} \nu_{\star}(x, y)=\cos x \cdot \delta(x-y)$. We believe that there is no cavity generating this measure; however, according to theorem 2.2 , there do exist cavities approximating it; that is, there exists a sequence of rough discs $B_{\varepsilon}$ such that $\nu_{B_{\varepsilon}}$ weakly converge to $\nu_{\star}$ (see Plakhov \& Gouveia (2007) for an explicit construction). One has $R_{T}\left[\nu_{\star}, \lambda\right]=3 \pi \lambda / 8, R_{L}\left[\nu_{\star}, \lambda\right]=-3 / 2$ and $R_{I}\left[\nu_{\star}, \lambda\right]=-3 \lambda / 2$. Thus, the longitudinal component of the resistance force does not depend on the angular velocity $\lambda$, while the transversal component and the moment of this force are proportional to $\lambda$.

(3) Rectangular cavity. The rough disc $B_{\varepsilon}$ is represented by the sequence of sets $B_{m}$ that are regular $m$-gons with $m$ congruent rectangles taken away (figure $8 a$ ). The width of the rectangles is much smaller than their 

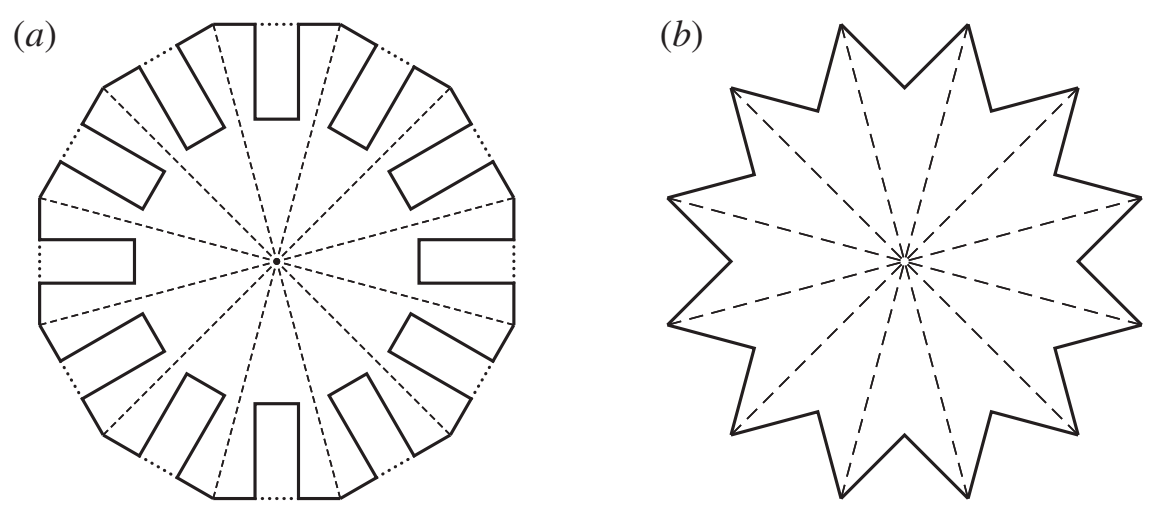

Figure 8. (a) A rough disc with rectangular cavities. (b) A rough disc with triangular cavities.

height $($ width $) /($ height of the rectangle $)=\varepsilon$. A smaller side of each rectangle is contained in a side of the polygon, besides |side of the rectangle|/|side of the polygon $\mid=1-\varepsilon$. Then $\nu_{B}=\nu_{\text {rect }}+o(1)$, where $\nu_{\text {rect }}=\left(\nu_{0}+\nu_{\star}\right) / 2$ and $o(1)$ stands for a measure weakly converging to zero as $\varepsilon \rightarrow 0^{+}$. One can easily calculate that $R_{T}\left[\nu_{\text {rect }}, \lambda\right]=3 \pi \lambda / 16, R_{L}\left[\nu_{\text {rect }}, \lambda\right]=-1.25$ and $R_{I}\left[\nu_{\text {rect }}, \lambda\right]=-3 \lambda / 4$.

(4) Triangular cavity. The sets $B_{m}$ representing the rough disc $B$ are regular $m$-gons with $m$ right isosceles triangles taken away (figure $8 b$ ). Then the measure $\nu_{B}=: \nu_{\nabla}$ has the following support (which looks like an inclined letter $\mathbf{H}):\{x+y=-\pi / 2: x \in[-\pi / 2,0]\} \cup\{y=x: x \in[-\pi / 4, \pi / 4]\} \cup$ $\{x+y=\pi / 2: x \in[0, \pi / 2]\}$. The density of this measure equals $\mathrm{d} \nu_{\nabla}(x, y)=\cos x$. $\left(\chi_{[-\pi / 2,-\pi / 4]}(x) \delta(x+y+\pi / 2)+\chi_{[-\pi / 4, \pi / 4]}(x) \cdot \delta(x-y)+\chi_{[\pi / 4, \pi / 2]}(x) \delta(x+y-\right.$ $\pi / 2))+|\sin x| \cdot\left(\chi_{[-\pi / 4,0]}(x) \delta(x+y+\pi / 2)-\chi_{[-\pi / 4, \pi / 4]}(x) \delta(x-y)+\chi_{[0, \pi / 4]}(x) \delta\right.$ $(x+y-\pi / 2))$. One has $\boldsymbol{R}\left[\nu_{\nabla}, 0^{+}\right]=(0 ;-\sqrt{2})^{\mathrm{T}}$ and $R_{I}\left[\nu_{\nabla}, 0^{+}\right]=0 ; \boldsymbol{R}\left[\nu_{\nabla}, 1\right]=$ $(1 / 4+3 \pi / 16 ; 3 \pi / 16-2)^{\mathrm{T}}$. The rest of the values are still unknown.

(5) Cavity realizing the product measure. Consider the measure $\nu_{\otimes}$ with the density $\mathrm{d} \nu_{\otimes}(x, y)=(1 / 2) \cos x \cos y \mathrm{~d} x \mathrm{~d} y$. Evidently, in this case, $\nu_{\otimes} \in \Pi^{\text {symm }}$. The angles of incidence and of reflection are statistically independent; so to speak, at the moment when the particle leaves the cavity, it completely 'forgets' its initial velocity. Here we have $R_{T}\left[\nu_{\otimes}, \lambda\right]=\left(10 \lambda+\lambda^{3}\right) \pi / 80$ for $0<\lambda \leq 1 ; R_{L}\left[\nu_{\otimes}, 1\right]=$ $-3 / 4-\pi / 5 \approx-1.378$ and $R_{I}\left[\nu_{\otimes}, \lambda\right]=-3 \lambda / 4$ for any $\lambda$. The remaining values are unknown.

The points in figure $7 a-d$ corresponding to cases $1-5$ are indicated by special symbols: $\nu_{0}$ is marked by a circle, $\nu_{\star}$ is marked by a diamond; $\nu_{\text {rect }}$, by an open square; $\nu_{\nabla}$, by a triangle and $\nu_{\otimes}$, by a circumscribed cross.

\section{Dynamics of a rough disc}

The motion of the spinning rough disc $B$ is determined by the values $R_{T}\left[\nu_{B}, \lambda\right]$, $R_{L}\left[\nu_{B}, \lambda\right]$ and $R_{I}\left[\nu_{B}, \lambda\right]$. For the sake of brevity, below we omit the fixed argument $\nu$ and write $R(\lambda)$ instead of $R[\nu, \lambda]$. Recall that the absolute value of the disc velocity is denoted by $v=|\boldsymbol{v}|$, and the angular velocity equals $\omega=\lambda v / r$. Denote by $\theta$ the angle the velocity makes with a fixed direction in an inertial frame of reference. 
Using equations (2.9) and (2.10), one rewrites the equations of motion (1.1) and (1.2) in the form

and

$$
\begin{aligned}
\frac{\mathrm{d} v}{\mathrm{~d} t} & =\frac{8 r \rho v^{2}}{3 M} R_{L}(\lambda), \\
\frac{\mathrm{d} \theta}{\mathrm{d} t} & =-\frac{8 r \rho v}{3 M} R_{T}(\lambda) \\
\frac{\mathrm{d}(\lambda v)}{\mathrm{d} t} & =\frac{8 r^{3} \rho v^{2}}{3 I} l R_{I}(\lambda) .
\end{aligned}
$$

Recall that $\beta=M r^{2} / I$ is the inverse relative moment of inertia. The special values that $\beta$ can take are: $\beta=1$, when the mass is concentrated near the disc boundary, and $\beta=2$, when the mass is uniformly distributed inside the disc. In the intermediate case, when the mass is arbitrarily (generally speaking, nonuniformly) distributed inside the disc, it holds $\beta \geq 1$.

With the change of variables $\mathrm{d} \tau=(8 \mathrm{r} \rho v / 3 M) \mathrm{d} t$, equations (4.1)-(4.3) are transformed into the following ones:

and

$$
\begin{aligned}
& \frac{\mathrm{d} \lambda}{\mathrm{d} \tau}=\beta R_{I}(\lambda)-\lambda R_{L}(\lambda), \\
& \frac{\mathrm{d} v}{\mathrm{~d} \tau}=v R_{L}(\lambda) \\
& \frac{\mathrm{d} \theta}{\mathrm{d} \tau}=-R_{T}(\lambda) .
\end{aligned}
$$

Denote by $s$ the path length of the disc; thus, $\mathrm{d} s / \mathrm{d} t=v$. One readily finds that $s$ is proportional to $\tau, s=(3 M / 8 r \rho) \tau$.

Below, we solve the system of equations (4.4)-(4.6) for cases 1-3 considered in $\S 3 b$. Next, we determine the dynamics numerically for some kinds of triangular cavities. A more detailed study of dynamics in the general case will be addressed elsewhere.

(1) Circle. One has $\mathrm{d} \lambda / \mathrm{d} \tau=-\lambda, \mathrm{d} v / \mathrm{d} \tau=-v$ and $\mathrm{d} \theta / \mathrm{d} t=0$; therefore the circle moves straightforward. Solving these equations, one obtains that its centre moves according to the equation $\boldsymbol{x}(t)=(3 \mathrm{M} / 8 \mathrm{r} \rho) \ln \left(t-t_{0}\right) \boldsymbol{e}+\boldsymbol{x}_{0}$, where $t_{0} \in \mathbb{R}$, $e \in S^{1}$ and $\boldsymbol{x}_{0} \in \mathbb{R}^{2}$ are constants. Thus, having started the motion at some moment, the circle passes a half-line during infinite time. This equation also implies that the motion cannot be extended to all $t \in \mathbb{R}$.

(2) Retroreflector. Here the system (4.4)-(4.6) takes the form

$$
\frac{\mathrm{d} \lambda}{\mathrm{d} \tau}=-\frac{3 \lambda(\beta-1)}{2}, \frac{\mathrm{d} v}{\mathrm{~d} \tau}=-\frac{3 v}{2} \text { and } \frac{\mathrm{d} \theta}{\mathrm{d} \tau}=-\frac{3 \pi \lambda}{8} .
$$

In the case $\beta=1$, one evidently has $\lambda=$ const. The disc moves along a circumference of radius $M /(\pi r \rho \lambda)$ in the direction opposite to the angular velocity of rotation: if the disc rotates counterclockwise, then its centre moves clockwise along the circumference. The radius of the circumference is proportional to the disc mass and inversely proportional to the relative angular velocity. The path length is proportional to the logarithm of time, $s(t)=(M / 4 r \rho) \ln \left(t-t_{0}\right)$. 
(a)

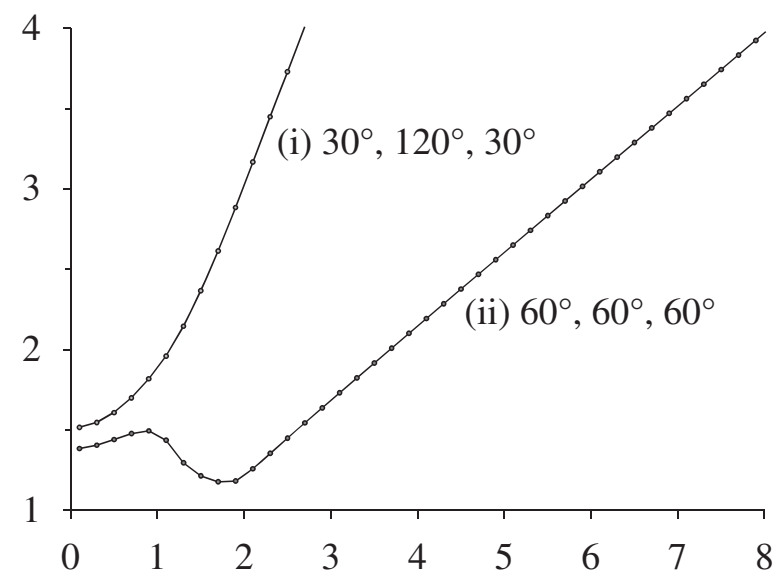

(b)

$\alpha(\lambda)$

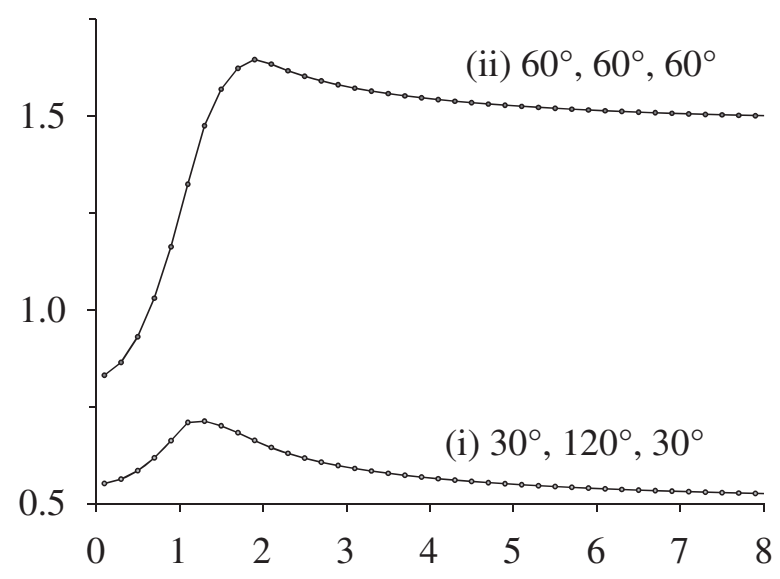

Figure 9. The functions $(a) g(\lambda)=\lambda R_{L}(\lambda) / R_{I}(\lambda)$ and $(b) \alpha(\lambda)=4 R_{T}(\lambda) / \lambda$ are shown for the triangular cavities with the angles (i) $30^{\circ}, 120^{\circ}, 30^{\circ}$ and (ii) $60^{\circ}, 60^{\circ}, 60^{\circ}$.

In the case $\beta>1$, we have $s(t)=(M / 4 r \rho) \ln \left(t-t_{0}\right), \quad \theta=\theta_{0}+$ const. . $\exp (-(\beta-1)(4 r \rho / M) s)$ and $\lambda=(4 / \pi)(\beta-1)\left(\theta-\theta_{0}\right)$. The path length once again depends logarithmically on the time, the relative angular velocity $\lambda$ converges to zero and the direction $\theta$ converges to a limiting value $\theta_{0}$; thus, the values $\lambda$ and $\theta$ are exponentially decreasing functions of the path length and are inversely proportional to the $(\beta-1)$ th degree of the time passed since a fixed moment. The trajectory of motion is a semibounded curve approaching an asymptote as $t \rightarrow+\infty$.

(3) Rectangular cavity. Equations of motion (4.4)-(4.6) in this case take the form $\mathrm{d} \lambda / \mathrm{d} \tau=-3 \lambda(\beta-5 / 3) / 4, \mathrm{~d} v / \mathrm{d} \tau=-5 v / 4$ and $\mathrm{d} \theta / \mathrm{d} \tau=-3 \pi \lambda / 16$. Solving these equations, one obtains $\tau=(4 / 5) \ln \left(t-t_{0}\right), \quad v=v_{0} \mathrm{e}^{-5 \tau / 4}, \quad \lambda=$ $\lambda_{0} \mathrm{e}^{3 \tau(5 / 3-\beta) / 4}$ and $\theta=\theta_{0}+\left(\pi \lambda_{0} / 4(\beta-5 / 3)\right) \mathrm{e}^{3 \tau(5 / 3-\beta) / 4}$. Thus, the path depends on $t$ logarithmically, and the relative angular velocity and the rotation angle are proportional to $\left(t-t_{0}\right)^{1-3 \beta / 5}$ and to $\exp ((2 r \rho(5-3 \beta) / 3 M) s)$.

If $\beta<5 / 3$, then $\lambda$ and $\theta$ tend to infinity, and the trajectory of the disc centre is a converging spiral. In the case $\beta>5 / 3, \lambda$ converges to zero, $\theta$ converges to a constant value and the trajectory is a semibounded curve approaching an asymptote as $t \rightarrow+\infty$. In the case $\beta=5 / 3, \lambda$ is constant, and the trajectory is a circumference of radius $2 M /(\pi r \rho \lambda)$.

Finally, we examine numerically some triangular cavities. It is helpful to denote $g(\lambda)=\lambda R_{L}(\lambda) / R_{I}(\lambda)$ and rewrite equation (4.4) in the form

$$
\frac{\mathrm{d} \lambda}{\mathrm{d} \tau}=-R_{I}(\lambda)(g(\lambda)-\beta)
$$

In figure $9 a$, the function $g(\lambda)$ is shown for two cases where the cavity is an isosceles triangle with the angles (i) $30^{\circ}, 120^{\circ}, 30^{\circ}$ and (ii) $60^{\circ}, 60^{\circ}, 60^{\circ}$. We see that $g(\lambda)$ monotonically increases in case (i) and has three intervals of monotonicity in case (ii). In both cases, $R_{I}(\lambda)<0$. This implies, in case (i), that the disc trajectory is a converging spiral, if $\beta<1.5$, and may take the form of a converging spiral or a curve approaching a straight line, depending on the initial conditions, if $\beta>1.5$. 


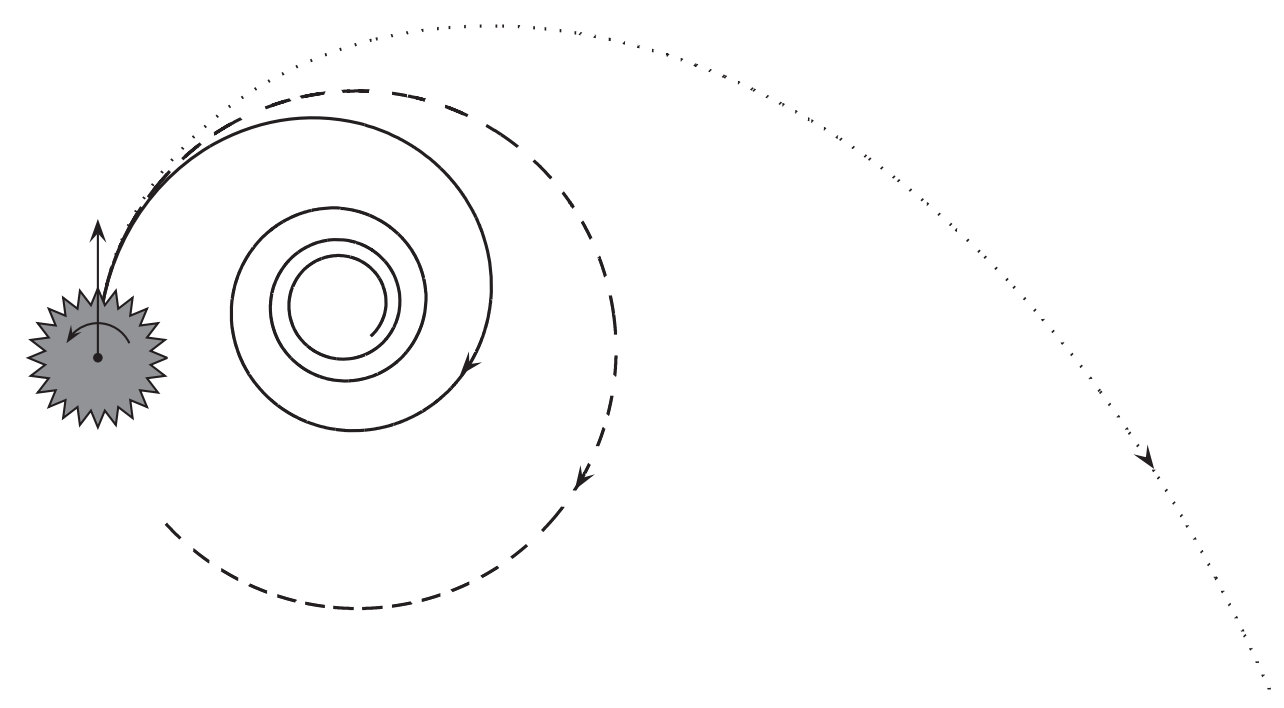

Figure 10. Three kinds of asymptotic behaviour of a rough disc with roughness formed by equilateral triangles and with $1.38<\beta<1.49$ : (I) converging spiral (solid line); (II) circumference (dashed line); (III) curve approaching a straight line (dotted line).

The disc behaviour is richer in case (ii) of the equilateral triangle. If $1.38<\beta<$ 1.49 , then three kinds of asymptotic behaviour may be realized, depending on the initial conditions: (I) the trajectory is a converging spiral, (II) the trajectory approaches a circumference, and (III) the trajectory approaches a straight line (figure 10). If $1.16<\beta<1.38$, only two asymptotic behaviours of types (I) and (II) are possible; if $\beta>1.49$, then the possible behaviours are (I) and (III); and if $\beta<1.16$, the asymptotic behaviour is always (I).

In the case of triangular cavities, as our numerical evidence shows, the function $g(\lambda)$ monotonically increases for $\lambda$ sufficiently large and $\lim _{\lambda \rightarrow+\infty} g(\lambda)=+\infty$. This implies that the trajectory is a converging spiral for appropriate initial conditions (namely, if the initial angular velocity is large enough). If, besides, $\beta$ is large enough (that is, the mass of the disc is concentrated near the centre), the trajectory may also be a curve approaching a straight line. If the function $g$ has intervals of monotone decrease (as for the case of the equilateral triangle), then the trajectory may also approach a circumference. The length of the disc path is always proportional to the logarithm of time.

\section{Conclusions and comparison with the previous works}

In our opinion, the inverse Magnus effect in highly rarefied media is caused by two factors:

(i) Non-elastic interaction of particles with the body. A part of the tangential component of the particles' momentum is transmitted to the body, resulting in creation of a transversal force.

(ii) Multiple collisions of particles with the body owing to the fact that the body's surface is not convex but contains microscopic cavities. 
In the papers by Borg et al. (2003), Ivanov \& Yanshin (1980), Wang (1972) and Weidman \& Herczynski (2004), the impact of factor (i) is studied. Moreover, the body is supposed to be convex and therefore factor (ii) is excluded from the consideration. In these papers, the force acting on a spinning body moving through a rarefied gas is calculated, and, additionally, the moment of this force slowing down the body's rotation is determined (Ivanov \& Yanshin 1980). The following shapes have been considered: a sphere, a cylinder (Ivanov \& Yanshin 1980; Weidman \& Herczynski 2004), convex bodies of revolution (Ivanov \& Yanshin 1980) and right parallelepipeds of regular polygon section (Weidman \& Herczynski 2004). The interaction of the gas particles with the body is as follows: a fraction $1-\alpha_{\tau}$ of the incident particles is elastically reflected according to the rule 'the angle of incidence is equal to the angle of reflection', while the remaining fraction $\alpha_{\tau}$ of the particles reaches thermal equilibrium with the body's surface, and is reflected as a Maxwellian (Wang 1972; Ivanov \& Yanshin 1980; Borg et al. 2003). In the paper by Weidman \& Herczynski (2004), a somewhat different model of interaction is considered, where the reflected particles acquire a fraction $\alpha_{\tau}$ of tangential momentum of the rotating body. The transversal force results from the tangential friction and acts on the body in the direction associated with the inverse Magnus effect. It is remarkable that for different models and different shapes of the body, the formula for the transversal force is basically the same. If the rotation axis is perpendicular to the direction of the body's motion, then this force equals

$$
\frac{1}{2} \alpha_{\tau} M_{g} \omega v,
$$

where $M_{g}$ is the mass of the gas displaced by the body, $\omega$ is the angular velocity of the body and $v$ is its translation velocity. (Note that in Borg et al. (2003), this formula appears in the limit of infinite heat conductivity or zero gas temperature.) Weidman \& Herczynski (2004) found that for parallelepipeds of regular $n$-gon section with $n$ odd, the transversal force depends on time, and the value of this force was determined. It is easy to calculate, however, that the time-averaged force is equal to equation (5.1).

In the present paper, in contrast, we concentrate on the study of factor (ii). We suppose that all collisions of particles with the body are perfectly elastic (that is, $\alpha_{\tau}=0$ ), and therefore there is no tangential friction. We restrict ourselves to the two-dimensional case and suppose that the body is a disc with small cavities on its boundary, or a rough disc. The Magnus effect is due to multiple reflections of particles in the cavities. We study here all logically possible cases of cavities. According to equation (2.9), the transversal force equals

$$
\frac{1}{2} \alpha(\lambda) M_{g} \omega v,
$$

where $\lambda=\omega r / v, M_{g}=\pi r^{2} \rho$ is the total mass of gas particles displaced by the body, $\alpha(\lambda)=\alpha(\lambda, \nu)=(16 / 3 \pi) R_{T}[\nu, \lambda] / \lambda$ and $\nu$ is the measure characterizing the shape of the cavities. The function $\alpha$ depends on both $\nu$ and $\lambda$. In particular, $\alpha$ varies between -0.409 and 2 for $\lambda=0.1$, between -0.378 and 2 for $\lambda=0.3$ and between -0.248 and 2 for $\lambda=1$. We conjecture that $\lim _{\lambda \rightarrow \infty} \inf _{\nu} \alpha(\lambda, \nu)=0$ and $\lim _{\lambda \rightarrow \infty} \sup _{\nu} \alpha(\lambda, \nu)=2$. The graphs of the function $\alpha(\lambda)$ with $\nu$ corresponding to triangular cavities with the angles (i) $30^{\circ}, 120^{\circ}, 30^{\circ}$ and (ii) $60^{\circ}, 60^{\circ}, 60^{\circ}$ 
are shown in figure $9 b$. We see that this function significantly depends on the velocity of rotation $\lambda$; in general, the variation of $\alpha(\lambda)$ with $\nu$ fixed can be more than twofold.

We conclude that the impact of both factors (i) and (ii) is unidirectional, and so they strengthen each other. Moreover, the formulas for the transversal force are similar; one should just substitute the function $\alpha(\lambda, \nu)$ for $\alpha_{\tau}$. We have seen that $\alpha(\lambda, \nu)$ can be significantly greater than 1 , while $\alpha_{\tau} \leq 1$. This can be just an artefact of our model being two-dimensional.

In a forthcoming work, we are planning to extend our consideration to the three-dimensional case and to media with a positive temperature. This seems to be more or less straightforward. A more challenging task would be studying the joint impact of non-elastic multiple collisions in the cavities. Also, it would be very interesting to study the effect in 'not so rarefied' media and find the critical value of density corresponding to reversal of the Magnus effect.

Apart from its physical meaning, studying the dynamics of a spinning rough disc (or, more generally, of a non-circular body) in a rarefied medium represents a nice mathematical problem, which originates in classical mechanics and has close connection with Newton's aerodynamic problem (Newton 1687). According to our numerical simulations, the most part of all possible roughnesses (93.6\% for $\lambda=1)$ correspond to the inverse Magnus effect, and only a small portion of them corresponds to the proper one. We know that roughnesses corresponding to the proper Magnus effect do exist, but have no idea how they should look and not one of such roughnesses has been found. Another interesting question concerns the description of admissible trajectories and is closely related to the associated problem of (vector-valued) Monge-Kantorovich OMT. In particular, the existence, in the same body, of a roughness corresponding to the proper Magnus effect for some values of $\lambda$ and to the inverse one for others would imply the existence of a rough disc with a strange zigzag trajectory.

This work was supported by the Centre for Research on Optimization and Control (CEOC) from the 'Fundação para a Ciência e a Tecnologia' (FCT), cofinanced by the European Community Fund FEDER/POCTI and by FCT: research project PTDC/MAT/72840/2006.

The authors are grateful to John Gough for useful comments on the text and to Gennady Mishuris for help in drawing figure 10.

\section{References}

Borg, K. I. \& Söderholm, L. H. 2008 Orbital effects of the Magnus force on a spinning spherical satellite in a rarefied atmosphere. Eur. J. Mech. B/Fluids 27, 623-631. (doi:10.1016/ j.euromechflu.2007.11.001)

Borg, K. I., Söderholm, L. H. \& Essénm, H. 2003 Force on a spinning sphere moving in a rarefied gas. Phys. Fluids 15, 736-741. (doi:10.1063/1.1541026)

Ivanov, S. G. \& Yanshin, A. M. 1980 Forces and moments acting on bodies rotating around a symmetry axis in a free molecular flow. Fluid Dyn. 15, 449. (doi:10.1007/BF01089985)

Mehta, R. D. 1985 Aerodynamics of sport balls. Annu. Rev. Fluid Mech. 17, 151-189. (doi:10.1146/ annurev.fl.17.010185.001055)

Newton, I. 1687 Philosophiae naturalis principia mathematica. London, UK: Streater.

Plakhov, A. 2004 Newton's problem of the body of minimum mean resistance. Sb. Math. 195, 1017-1037. (doi:10.1070/SM2004v195n07ABEH000836) 
Plakhov, A. 2009a Billiards and two-dimensional problems of optimal resistance. Arch. Ration. Mech. Anal. 194, 349-382. (doi:10.1007/s00205-008-0137-1)

Plakhov, A. $2009 b$ Billiard scattering on rough sets: two-dimensional case. SIAM J. Math. Anal. 40, 2155-2178. (doi:10.1137/070709700)

Plakhov, A. \& Gouveia, P. 2007 Problems of maximal mean resistance on the plane. Nonlinearity 20, 2271-2287. (doi:10.1088/0951-7715/20/9/013)

Plakhov, A. \& Tchemisova, T. 2009 Force acting on a spinning rough disk in a flow of noninteracting particles. Dokl. Math. 79, 132-135. (doi:10.1134/S1064562409010396)

Prandtl, L. 1926 Application of the 'Magnus Effect' to the wind propulsion of ships. Die. Naturwiss. 13, 93-108 [NACA-TM-367].

Rachev, S. T. \& Rüschendorf, L. 1998 Mass transportation problems. Vol. 1: Theory. Vol. II: Applications. Probability and its Applications (New York), xxvi+508pp. New York, NY: Springer-Verlag.

Rubinov, S. I. \& Keller, J. B. 1961 The transverse force on a spinning sphere moving in a viscous fluid. J. Fluid Mech. 11, 447-459. (doi:10.1017/S0022112061000640)

Villani, C. 2003 Topics in optimal transportation. Graduate Studies in Mathematics, no. 58, xvi+370pp. Providence, RI: American Mathematical Society.

Wang, C.-T. 1972 Free molecular flow over a rotating sphere. AIAA J. 10, 713. (doi:10.2514/ 3.50192)

Weidman, P. D. \& Herczynski, A. 2004 On the inverse Magnus effect in free molecular flow. Phys. Fluids 16, L9-L12. (doi:10.1063/1.1633265) 